\title{
Agrarian Research Cooperation Between the Institutes of European and Developing Countries

\author{
Luis Alfaro Cardoso
}

Abstract: The author discusses the framework guidelines believed to support efficient conditions of scientific and technical cooperation with developing countries. Reference is made to priorities of project conception concerning the interest of industrial and developing countries.

\section{INTRODUCTION}

Our concern in research in the developing areas lies primarily in the agrarian field. In our activities we have the collaboration of the veterinary faculty from the Lisbon Technical University. We are attached to countries which have been or are still suffering considerable social and productive disruption due to war situations. Thus, our efforts, together with the efforts of those countries' scientific and rural extension staffs, go to the rehabilitation of the production and research framework. This is often a difficult task, for its accomplishment requires a practical realization of a sound concept of cooperation between countries. To the developing areas, this relationship means the building up of research programmes closely related to problems concerning their agrarian productions and to the reinforcement of their own research and education institutions. For the developed countries, the successful cooperation activities prepare the way for industrial, trading and education institutions.

\section{RESEARCH AND DEVELOPMENT (R\&D) PROJECTS}

Bearing in mind this cooperation concept, we consider of great importance the criteria with which the reference terms of research and development (R\&D) projects are defined. In our opinion, developing countries should have a sharp identification of the areas in need of research programmes, including activities concerning agrarian planning, work organization, trading and storing of agriculture products, etc.

These $R \& D$ projects should always take into consideration the social and economic conditions existing in the countries for which they are designed. Often the background in which the projects take place is one of depression of the productive systems; those projects have as a final goal the rehabilitation and improvement of those systems. For this reason, research project protocols, in our view, should always contain an extension and development component to be used as an evaluating instrument, so as to judge to what extent the projects are achieving or will achieve their aims.

We believe that the levels and technological means to be utilized in the research projects should be completely executed in the countries receiving the projects. The economic feasibility of the project equipment should be assured, even after the completion of the projects.

The building up of a solid national technical staff should, we think, always be a basic component of research programmes, so as to guarantee that, with project evolution, local professionals will be able to deal with all methodology used in the project, including mastering the laboratory equipment belonging to the project.

The design of cooperation projects convenient to the devel- oped countries is usually easy to define. The projects correspond to scientific areas in which they have accumulated work and experience, preferably overlapping industrial or trading interests. We think that it is under these circumstances, in which their scientific and economical interests are complemented and respected, that the developed countries can commit themselves to the R\&D projects in positive and favourable conditions, as in tropical areas.

We believe, however, that some work can be done in order to make possible the accumulated and current work in European tropical research institutes. A way of doing it could be by drawing up agreements between them so as to bring up the possibility of complementary scientific areas within easy reach of research teams, made up of professionals from various countries. In our view, the planning of fundamental science areas among European tropical research institutions will enhance the rentability of human and equipment resources. This kind of action could lead to European cooperation in activating regional scientific institutes in tropical areas, with special attention to Africa. In these institutes, which could have action ranges spanning several countries, specific working fields could be developed according to regional priorities.

The fact that these would be localized in the areas where the objectives of the R\&D programmes are found would contribute, in our opinion, to a correct tactic and strategic definition of the research projects. It would also increase the quality of the experience exchange between African and European experts and technicians. It would help to bring to African countries many of the tropical agrarian undergraduate and postgraduate courses that today are attended by African students in Europe. The evolvement of the developing countries' universities in the R\&D programmes seems important to us because the programmes offer students an early start in the linkage between theoretical and practical knowledge.

\section{CONCLUSION}

In brief, we believe that the rentability of research cooperation has a direct proportion to the human and capital investment strictly done in the developing countries. A large number of European countries accumulated a considerable amount of valuable scientific work during and after their colony-holding years. In many African countries, scientists and technicians have produced, in spite of great adversity, significant results in research and development. The systematic use and deepening of this knowledge is of vital importance to both developed and underdeveloped countries. This is to be understood by people who are responsible for drawing the guidelines of cooperation schemes, so as to avoid the frequent shortsightedness of policy-makers who often replace solid and locally useful projects with colourful and spectacular ephemerality. 